\title{
Sensitivity Analysis of Flutter of A Two-Degree of Freedom Linear Aeroelastic System
}

\author{
Riccy Kurniawan \\ Department of Mechanical Engineering, Atma Jaya Catholic University of Jakarta \\ Email: riccy.kurniawan@atmajaya.ac.id
}

\begin{abstract}
This paper deals with the problem of the aeroelastic stability of a typical aerofoil section with two degrees of freedom induced by unsteady aerodynamic loads. A method is presented to model the unsteady lift and pitching moment acting on a two dimensional typical aerofoil section, operating under attached flow conditions in an incompressible flow. Starting from suitable generalisations and approximations to aerodynamic indicial functions, the unsteady loads due to an arbitrary forcing are represented in a state-space form. From the resulting equations of motion, the flutter speed is computed through stability analysis of a linear statespace system. The sensitivity analysis of the aeroelastic stability boundaries to the structural parameter is evaluated. The results show that the parameter with the greatest influence on flutter speed is the center of gravity.
\end{abstract}

Keywords: Incompressible, flutter, typical aerofoil section.

\section{INTRODUCTION}

Flutter is the dynamic aeroelasticity phenolmenon whereby the inertial forces can modify the behavior of a flexible system so that energy is extracted from the incoming flow. The flutter or critical speed $V_{F}$ represents the neutral stability boundary: oscillations are stable at speeds below it, but they become divergent above it.

Theodorsen [1] obtained closed-form solution to the problem of an unsteady aerodynamic load on an oscillating aerofoil. This approach assumed the harmonic oscillations in in-viscid and incompressible flow subject to small disturbances. Wagner [2] obtained a solution for the so-called indicial lift on a thin-aerofoil undergoing a transient step change in angle of attack in an incompressible flow. The indicial lift response makes a useful starting point for the development of a general time domain unsteady aerodynamics theory. A practical way to tackle the indicial response method is through a state-space formulation in the time domain, as proposed, for instance by Leishman and Nguyen [3].

The main objective of this paper is to investigate the aeroelastic stability of a typical aerofoil section with two degrees of freedom induced by the unsteady aerodynamic loads defined by the Leishman's state-space model. The sensitivity analysis is discussed to examine theinfluence of the structural parameters of the typical aerofoil section on theflutter margin

\section{RESEARCH METHOD}

The mechanical model under investigation is a two-dimensional typical aerofoil section in a horizontal flow of undisturbed speed $V$, as shown in Figure 1. Its motion is defined by two independent degrees of freedom, which are selected to be the vertical displacement (plunge), $h$, and the rotation (pitch), $a$.

The equations of motion for the typical aerofoil section have been derived in many textbooks of aeroelasticity, and can be expressed in nondimensional form as

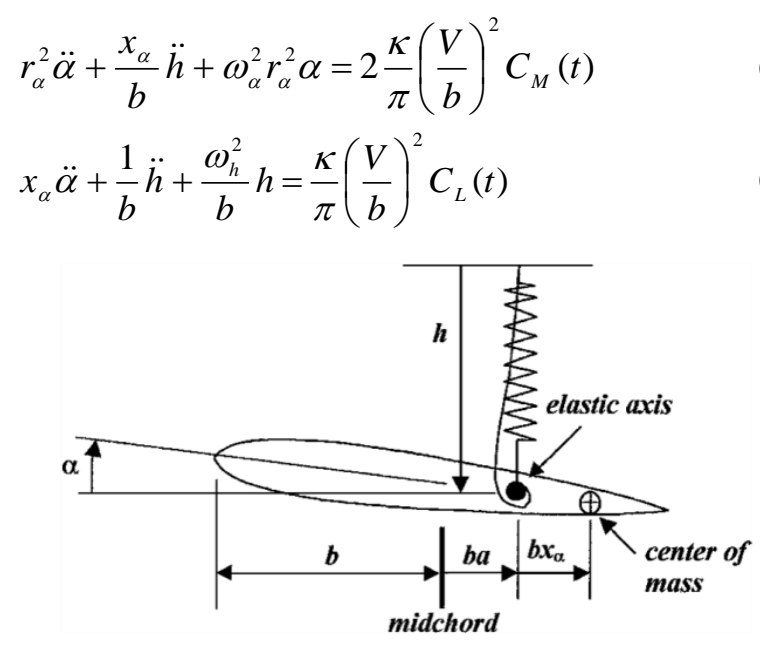

Figure 1. A Typical Aerofoil Section with Two Degrees of Freedom 
where $C_{M}(t)$ and $C_{L}(t)$ denote the coefficients of the aerodynamic forces corresponding to pitching moment and lift, respectively. For a general motion, where an aerofoil of chord $c=2 b$ is undergoing a combination of pitching and plunging motion in a flow of steady velocity $V$, Theodorsen[1] obtained the aerodynamic coefficients

$$
\begin{aligned}
C_{M}(t)= & -\frac{\pi}{2 V^{2}}\left[\left(\frac{1}{8}+a^{2}\right) \ddot{\alpha}-a b \ddot{h}\right]+ \\
& \pi\left(a+\frac{1}{2}\right) C(k) \alpha_{q s}-\frac{\pi}{2 V^{2}}\left[V\left(\frac{1}{2}-a\right) \dot{\alpha}\right] \\
C_{L}(t)= & \frac{\pi b}{V^{2}}(V \dot{\alpha}+\ddot{h}-b a \ddot{\alpha})+2 \pi C(k) \alpha_{q s}
\end{aligned}
$$

Theodorsen'sfunction is a complex-valued transfer function which depends on the reduced frequency $k$, where

$k=\frac{\omega b}{V}$

$\alpha_{q s}$ represents a quasi-steady aerofoil angle of attack, i.e.

$\alpha_{q s}=\frac{\dot{h}}{V}+\alpha+b\left(\frac{1}{2}-\alpha\right) \frac{\dot{\alpha}}{V}$

The indicial response method is the response of the aerodynamic flow field to a step change in a set of defined boundary conditions such as a step change in aerofoil angle of attack, in pitch rate about some axis, or in a control surface deflection (such as a tab of flap). If the indicial aerodynamic responses can be determined, then the unsteady aerodynamic loads due to arbitrary changes in angle of attack can be obtained through the superposition of indicial aerodynamic responses using the Duhamel's integral.

Assuming two-dimensional incompressible potential flow over a thin aerofoil, the circulatory terms in Equations (3) and (4) can be written as

$$
C(k) \alpha_{q s}=\alpha_{q s}(0) \phi_{w}(s)+\int_{0}^{s} \frac{d \alpha_{q s}}{d t} \phi_{w}(s-t) d t
$$

where $s$ is the non-dimensional time, given by

$s=\frac{1}{b} \int_{0}^{t} V d t$

$\phi_{w}$ is Wagner's function, which accounts for the influence of the shed wake, as does Theodorsen's function. In fact, both Wagner's and Theodorsen's function represents a Fourier transform pair. Wagner's function is known exactly in terms of Bessel functions (see [2] for details), but for practical implementation it is useful to represent it approximately. One of the most useful expressions is an exponential of the form

$$
\phi_{w}(s) \approx 1-A_{1} e^{-b_{1} s}-A_{2} e^{-b_{2} s}
$$

One exponential approximation is given by R.T. Jones [4] as

$$
\phi_{w}(s) \approx 1-0.165 e^{-0.0455 s}-0.335 e^{-0.3 s}
$$

The state-space equations describing the unsteady aerodynamics of the typical aerofoil section with two degrees of freedom can be obtained by direct application of Laplace transforms to the indicial response as

$\left[\begin{array}{l}\dot{z}_{1} \\ \dot{z}_{2}\end{array}\right]=\left[\begin{array}{cc}0 & 1 \\ -b_{1} b_{2}\left(\frac{V}{b}\right)^{2} & -\left(b_{1}+b_{2}\right) \frac{V}{b}\end{array}\right]\left[\begin{array}{l}z_{1} \\ z_{2}\end{array}\right]+\left[\begin{array}{l}0 \\ 1\end{array}\right] \alpha_{q s}$

with the output

$C(k) \alpha_{q s}=\left[\frac{b_{1} b_{2}}{2}\left(\frac{V}{b}\right)^{2} \quad\left(A_{1} b_{1}+A_{2} b_{2}\right)\left(\frac{V}{b}\right)\right]\left[\begin{array}{l}z_{1} \\ z_{2}\end{array}\right]+\frac{1}{2} \alpha_{q s}$

The indicial approach and the state-space formulation lead to a dynamic matrix that governs the behaviour of the system and enables future prediction. The analysis of flutter in this case is straightforward and it can be performed in the frequency domain, since the eigenvalues of the dynamic matrix directly determine the stability of the system. If, for a given velocity, any of the eigenvalues has a zero real part, the system is neutrally stable, i.e., it defines the flutter onset.

\section{RESULTS AND DISCUSSION}

In this section, the stability analysis of the state-space aeroelastic equation is presented. The results have been validated against published and experimental results. The sensitivity analysis is discussed to examine the influence of the structural parameters of the typical aerofoil section on the flutter onset.

\section{Validation against Published Results}

Theodorsen and Garrick [5] presented a graphical solution of the flutter speed of the twodimensional aerofoil for the flexture-torsion case. In order to validate the present model, a flutter speed computation is performed with varying combinations of aeroelastic parameters, as used by Theodorsen and Garrick, as shown in Table 1.

Table 1. Aeroelastic Parameters for the Validation

\begin{tabular}{ccccc}
\hline Case & $x_{\alpha}$ & $\kappa$ & $a$ & $r_{\alpha}^{2}$ \\
\hline $\mathrm{a}$ & 0.2 & $1 / 3$ & -0.4 & 0.25 \\
$\mathrm{~b}$ & 0.2 & $1 / 4$ & -0.2 & 0.25 \\
$\mathrm{c}$ & 0 & $1 / 5$ & -0.3 & 0.25 \\
$\mathrm{~d}$ & 0.1 & $1 / 10$ & -0.4 & 0.25 \\
\hline
\end{tabular}

Figure 2. shows the comparison of the flutter margin from Theodorsen and Garrick's work with the present computation. In the graph, non-dimensional flutter speed $V_{F} *$ is presented as a function of the frequency ratio $\omega_{h} / \omega_{\alpha}$. As can be seen, the present method provides a good agreement with the 
published figures only for low frequency ratios. In fact, as the ratio approaches unit value, the actual curve drifts to generally lower speeds.

This discrepancy is probably due to numerical inaccuracies in the curves presented in the original work. Zeiler [6] found a number of erroneous plots in the reports of Theodorsen and Garrick and provided a few corrected plots. In order to verify the validity of Zeiler's statement, the numerical computation of the flutter speed is conducted using the aeroelastic parameters used by Zeiler.

Figure 3 shows some of the results obtained by Zeiler, compared to the figures obtained by Theodorsen and Garrick and those obtained using the present state-space method. As can be observed, the agreement with Zeiler is very good, whereas Theodorsen and Garrick's results deviate considerably. It confirms the validity of Zeiler's statement and provides evidence of the validity of the results obtained here.

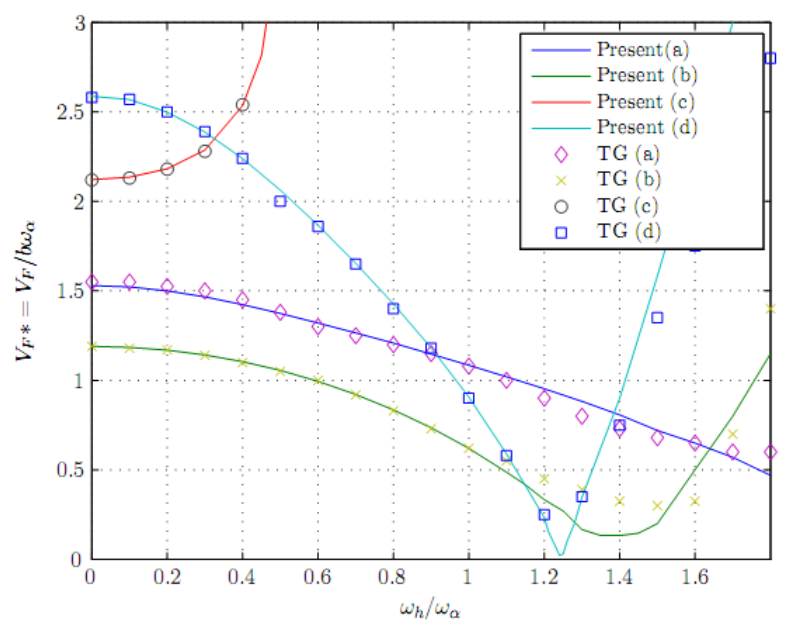

Figure 2. Comparison of Flutter Boundaries from Theodorsen and Garrick [5] with Present Computations

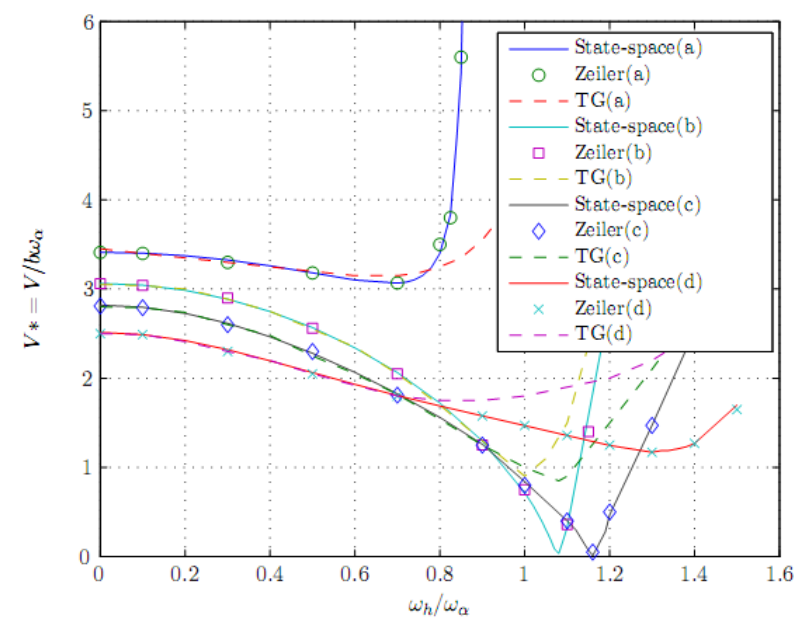

Figure 3. Comparison of the Flutter Boundaries from Zeiler [6], and Theodorsen and Garrick [5] with the Present Computations. The parameters used are $\alpha=-0.3, \kappa=0.05, r_{\alpha}^{2}=0.25, b=0.3$ (a) $x_{\alpha}=0$ (b) $x_{\alpha}$ $=0.05$ (c) $x_{\alpha}=0.1$ (d) $x_{\alpha}=0.2$

\section{Validation Against Experimental Data}

Experimental flutter results for a typical aerofoil section with two degrees of freedom were investigated by Sivakumar [7]. Pitch and plunge are provided by a set of eight linear springs. The set-up was clamped to the $5 \times 4$ Donald Campbell wind tunnel. The airspeed was gradually increased until the onset of flutter. The parameter values used in the experimental study are shown in Table 2.

Table 2. Structural Properties of an Aerofoil in the Experimental Study

\begin{tabular}{cccccccc}
\hline Parameter & $\boldsymbol{x}_{\boldsymbol{a}}$ & $\boldsymbol{\kappa}$ & $a$ & $\boldsymbol{r}_{\boldsymbol{a}}$ & $b$ & $\boldsymbol{\omega}_{\boldsymbol{a}}$ & $\boldsymbol{\omega}_{\boldsymbol{h}}$ \\
\hline Value & 0.00064 & 0.0157 & -0.1443 & 0.4730 & 0.05 & 61.5637 & 8.8468 \\
\hline
\end{tabular}

The non-dimensional flutter speed resulting from the present computation flutter analysis is $V_{\text {nom }} *=4.31$ and that from the experimental study is $V_{\text {exp }} *=4.04$. The comparison shows that the value of the experimental flutter speed is therefore $6.26 \%$ smaller than the numerical flutter speed. This is may be due to the error and uncertainty that is well accepted to occur in experimental studies, and which has affected the flutter speed measurement. Nevertheless, the flutter speed obtained in the experiments agrees with the numerical results fairly well.

\section{Parametric Study}

The flutter boundaries of a typical aerofoil section subjected to unsteady aerodynamic forces are affected by a number of structural parameters such as the location of the centre of gravity, radius of gyration, elastic axis position and ratio of mass. Therefore, we need to examine the relationships between these parameters and the flutter boundaries in order to optimise the design.

Theodorsen and Garrick [5] perform a theoretical survey of the effect of the flutter parameters for the flexture-torsion case. The present parametric study uses a similar algorithm of the work of Theodorsen and Garrick by varying one parameter at a time and keeping all the others constant. The reference values which are used in this parametric study are $a=0.2, b=0.3, x_{a}=0.1, \kappa=0.05$ and $r_{a}{ }^{2}=0.25$. The flutter speeds are computed over a range of flextural-torsion frequency ratios, and plotted as dimensionless flutter speed, normalised with respect to the uncoupled natural torsional frequency and the half chord length.

From Figure 4a it can be observed as $x_{a}$ increases, that the maximum value of the flutter speed is reduced and the dip is slightly displaced rightwards in the graph. 


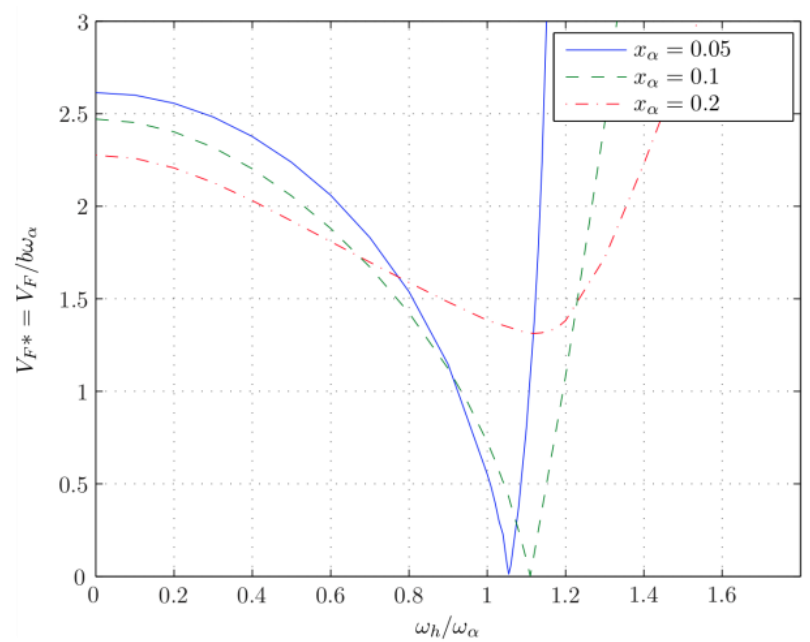

(a)

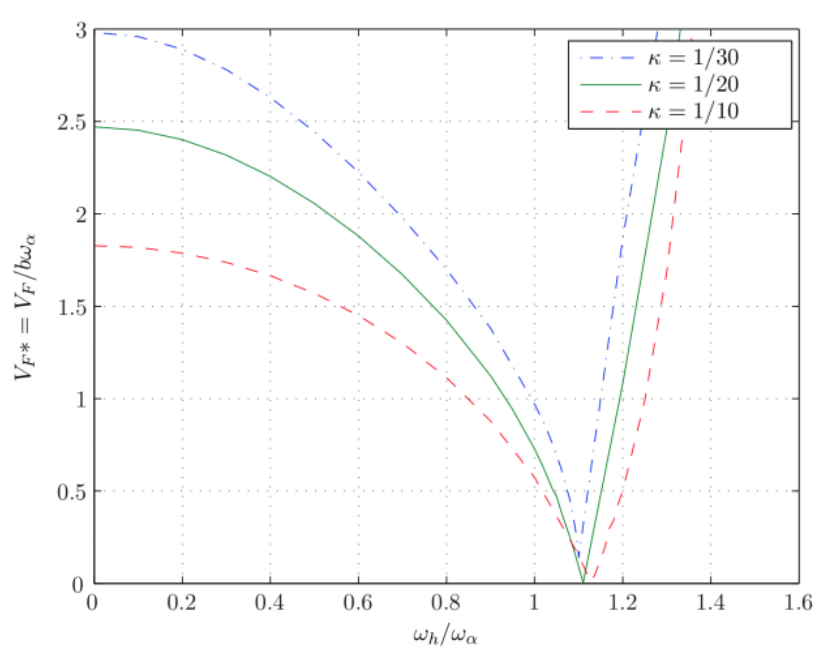

(c)

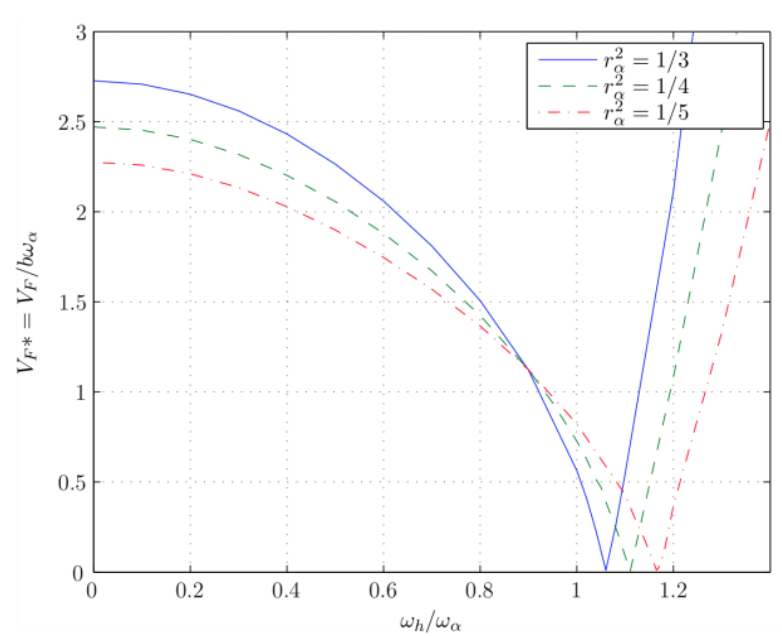

(b)

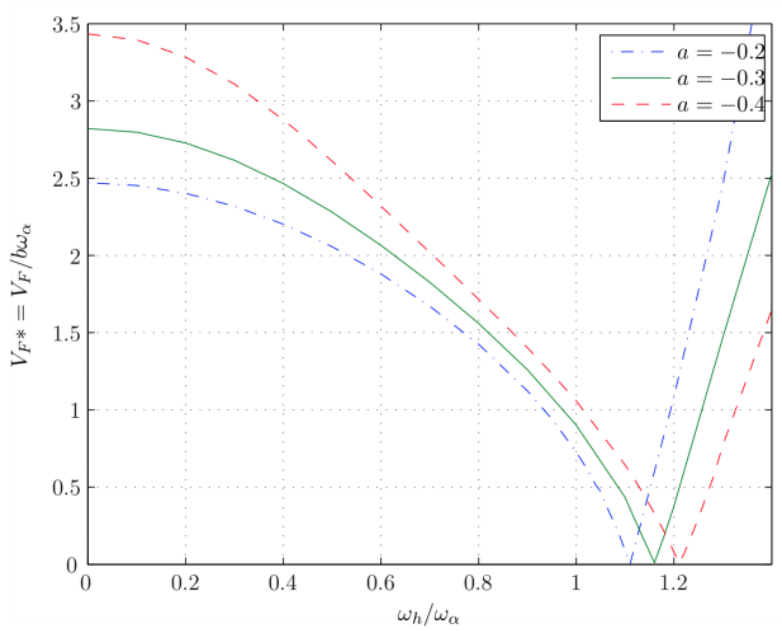

(d)

Figure 4. Influence of (a) centre of gravity (b) radius of gyration (c) mass density ratio (d) elastic axis position on flutter speed.

Figure $4 \mathrm{~b}$ shows the flutter speed increases with an increase in the radius of gyration. The dip is slightly displaced leftwards as radius of gyration is increased. From Figure 4 (c) it can be deduced that as $\kappa$ is increased, the maximum value of the flutter speed is significantly reduced. Figure 4 (d) shows how the flutter speed versus the frequency ratio varies with the elastic axis position, $a$. By moving the elastic axis aft, the flutter speed is decreased and the dip displaced leftwards.

\section{CONCLUSIONS}

A model to determine the flutter onset of a twodimensional typical aerofoil section has been implemented and then validated. A traditional aerodynamic analysis, based on Theodorsen's theory and Leishman's state-space model was used. The validation was performed, firstly, by solving Theodorsen and Garrick's problem for the flexture-torsion flutter of a two-dimensional typical aerofoil section. The stability curves obtained are in close agreement with the results reported by more recent solutions of the same problem, whereas the original figures from Theodorsen and Garrick are found to be biased, as was previously reported by Zeiler. Secondly, validation with experimental data was conducted and the results showed a fairly close agreement.

The parametric study of flutter speeds to structural parameters was evaluated by changing one parameter at a time, while keeping all others constant. Knowledge of the functional dependency of the flutter speed on each parameter is essential in order to obtain sufficient accuracy in determining the important parameters and preventing wasting time on those with less influence. It is shown here that the most important parameter is the location on the centre of gravity. 


\section{REFERENCES}

1. Theodorsen, T., General theory of aerodynamic instability and the mechanism of flutter, NACA Report number: 496, 1934.

2. Wagner, H., Uber die Entstehung des dynamischenAuftriebes von Tragfliigeln, Zietschrift fur AngewandteMathematik und Mechanick, 5(1), 17-35, 1925.

3. Leishman, J.G. and Nguyen, K.Q. State-space representation of unsteady airfoil behavior, AIAA Journal, 28(5), 863-844, 1989.
4. Jones, R.T., The unsteady lift of a wing of finite aspect ratio, NACA report number: 681, 1940.

5. Theodorsen, T. and Garrick, I.E., Mechanism of flutter: a theoretical and experimental investigation of flutter problem, NACA Report number: 685, 1938.

6. Zeiler, T.A., Results of Theodorsen and Garrick revisited, Journal of Aircraft, 37(5), 918-920, 2000.

7. Sivakumar, S., Aeroelastic characteristics of a 2DOF aerofoil system, MSc Project Department of Aeronautics Imperial College London., 2009. 